\title{
Crizotinib plus radiotherapy in brain oligoprogressive NSCLC ROS1 rearranged and PD-L1 strong
}

\author{
Mario Occhipinti ${ }^{1}$, Rosa Falcone ${ }^{1,2}$, Concetta Elisa Onesti ${ }^{1,2}$, Andrea Botticelli ${ }^{2}$, Federica Mazzuca ${ }^{1,2}$, \\ Paolo Marchetti ${ }^{1,2,3}$, Salvatore Lauro ${ }^{1,2}$ \\ ${ }^{1}$ Medical Oncology Unit, Sant' Andrea Hospital, Rome, Italy; ${ }^{2}$ Department of Clinical and Molecular Medicine, "Sapienza” University of Rome, \\ Rome, Italy; ${ }^{3}$ Istituto Dermopatico dell'Immacolata-IRCCS, Rome, Italy \\ Correspondence to: Concetta Elisa Onesti. Medical Oncology Unit, Sant' Andrea Hospital, Via di Grottarossa 1035-1039, 00189 Rome, Italy. \\ Email: elisaonesti@gmail.com.
}

\begin{abstract}
ROS1+ patients represent a unique molecular subset of non-small cell lung cancer (NSCLC). Early phase clinical trials have shown a high response rate to crizotinib in these patients. We describe a case of an 18 years old woman, never smoker, with NSCLC ROS1+ and miliary brain metastases treated with crizotinib and radiotherapy. From October 2014 to June 2015 the Patient was treated with crizotinib. The first intracranial time to progression (IT-TTP) occurred after 7 months; the patient underwent stereotactic radiosurgery (SRS) and continued TKI treatment. The second IT-TTP appeared after 16 months. A continued response in the chest was observed for all the 23 months of crizotinib treatment. At the progression, we assessed programmed death ligand 1 (PD-L1) expression by immunohistochemistry, that resulted highly expressed. Our report indicates that the integration of crizotinib with local treatments should be considered in ROS1 NSCLC patients experiencing oligometastatic progression. Moreover, this case is an example of PD-L1 strong in oncogene addicted patients.
\end{abstract}

Keywords: Crizotinib; non-small cell lung cancer (NSCLC); oligoprogression; programmed death ligand 1 (PD-L1); radiotherapy; ROS1

Submitted Apr 04, 2017. Accepted for publication Aug 04, 2017.

doi: $10.21037 /$ jtd.2017.09.74

View this article at: http://dx.doi.org/10.21037/jtd.2017.09.74

\section{Introduction}

Treatment approaches for non-small cell lung cancer (NSCLC) have been advanced in recent years by identification of distinct, moleculary-defined subsets of patients, called oncogene addicted, that derive benefit from target therapy. Recently genetic alteration in ROS1 was identified in patients with NSCLC. ROS1 is a gene located on chromosome 6 (6q22), where it encodes an orphan tyrosine kinase receptor of the insulin receptor family. Chromosomal rearrangements involving the ROS1 gene consist on the fusion with other genes (CD74, TPM3, SDC4, EZR, LRIG3, FIG), leading to constitutive kinase activity. Until now, at least 12 ROS1 fusion variants have been identified in NSCLC (1).

Bergethon et al. detected ROS1 rearrangements in
18 (1.7\%) of 1,073 NSCLC patients using fluorescent in situ hybridization (FISH) and suggested that ROS1 rearrangements define a unique molecular subset of lung cancer with distinct clinical characteristics (2). Zhu et al., in a meta-analysis of 18 studies, including 9,898 NSCLC patients, revealed a significantly higher rate of ROS1 fusion gene in: female $(\mathrm{OR}=1.54,95 \% \mathrm{CI}: 1.02-2.34, \mathrm{P}=0.042)$, patients without smoking history $(\mathrm{OR}=3.27,95 \% \mathrm{CI}$ : 1.44 7.45, $\mathrm{P}=0.005)$, adenocarcinomas $(\mathrm{OR}=10.24,95 \% \mathrm{CI}$ : 5.13-20.40, $\mathrm{P}<0.001)$ and patients with an advanced clinical stage III-IV (OR =2.57, 95\% CI: 1.78-3.71, P<0.001) (3).

Due to the high degree of homology within the tyrosine kinase domains of $A L K$ and ROS1, ALK inhibitors were tested in ROS1-positive cell lines and tumours, showing an inhibitory activity.

Based on this preclinical data, the phase I PROFILE 

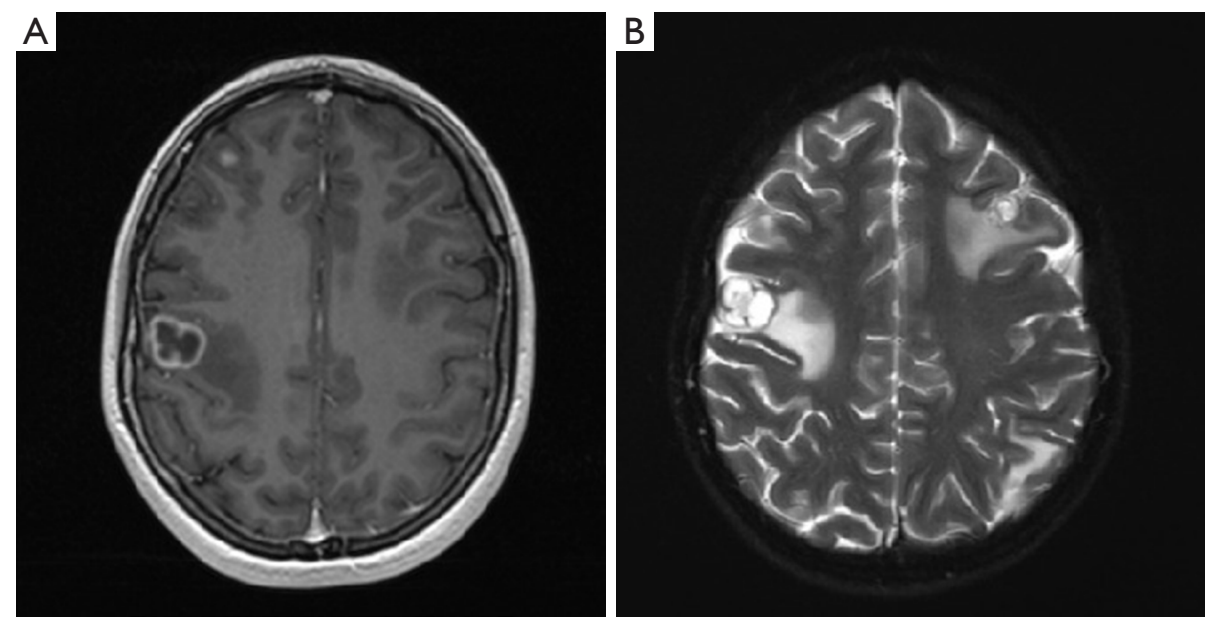

Figure 1 (A) and (B) show the baseline brain magnetic resonance imaging (MRI), October 2014.

trial of crizotinib [phase 1 open-label, single-arm, doseescalation study with an expansion cohort evaluating the safety, pharmacokinetic and pharmacodynamic of crizotinib (PF-02341066)] was amended to include a ROS1-positive cohort. Ou et al. reported the results of this phase I trial in 36 patients, showing $61 \%$ of overall response rate (ORR), suggesting the high clinical efficacy of Crizotinib in this subset of patients [Ou SH, Kim DW, Camidge DR, et al. Crizotinib therapy for patients with advanced ROS1 rearranged non-small cell lung cancer. 7 Thorac Oncol 2013;8(suppl 2):S295].

The efficacy of Crizotinib in NSCLC patients harbouring ROS1 translocation was demonstrated by Shaw et al. in a phase I trial (4). Currently crizotinib is approved by the Food and Drug Administration and by the European Medicines Agency for the treatment of ROS1-positive NSCLCs defined by FISH.

We describe the case of a young patient affected by a NSCLC ROS1 positive and programmed death ligand 1 (PD-L1) strong with central nervous system (CNS) metastases that benefited from crizotinib treatment.

\section{Case presentation}

On October 2014 an 18-year-old Caucasian female, never smoker, was admitted to the hospital with severe dyspnoea, cough and high fever. The total body CT scan revealed a mass in the right lower lobe with several mediastinal and supraclavicular adenopathy, lymphangitis, massive pulmonary embolism and miliary brain metastases, confirmed by a brain magnetic resonance imaging (MRI) (Figure 1A,B). The histological examination, obtained by a supraclavicular lymph-node biopsy, showed a NSCLC mucinous adenocarcinoma TTF1+, CK20-, KRAS wt, EGFR wt, ALK not rearranged and with ROS1 rearrangement detected by FISH. The patient underwent a whole brain radiotherapy (WBRT) and on November 2014 she started crizotinib $250 \mathrm{mg}$ twice daily as first line treatment. Since the first month of treatment the patient showed a significant clinical benefit and the subsequent total body CT scan, performed on January 2015, showed a partial response on the lung mass $(80 \%$ reduction in the thoracic lesion, $30 \mathrm{~mm} \times 20 \mathrm{~mm} v s .60 \mathrm{~mm} \times 57 \mathrm{~mm}$ ) and a complete response of brain metastases (Figure $2 A, B$ ). During the first months of treatment the patient experienced diarrhoea G1, nausea G2, neutropenia G1 and peripheral edema. All toxicities were completely solved after eight cycles of treatment. After 7 months of treatment, in June 2015, a brain MR revealed oligoprogression of the disease (Figure $3 A, B)$. The patient performed a stereotactic radiosurgery (SRS) of five brain metastases and continued crizotinib treatment, maintaining a good performance status and quality of life. On October 2016, due to seizure and speech impairments, the patient was admitted to the emergency department and a brain MRI evidenced further disease progression. Conversely, the total body CT scan revealed partial response in the chest (Figure 4). Crizotinib was permanently discontinued after 23 months of treatment because of brain progression. The patient underwent SRS on November 2016.

Subsequently, we assessed PD-L1 tumor expression with the use of PD-L1 IHC 22C3 pharmDx assay (Dako North America, Carpinteria, USA), with evidence of a PD-L1 

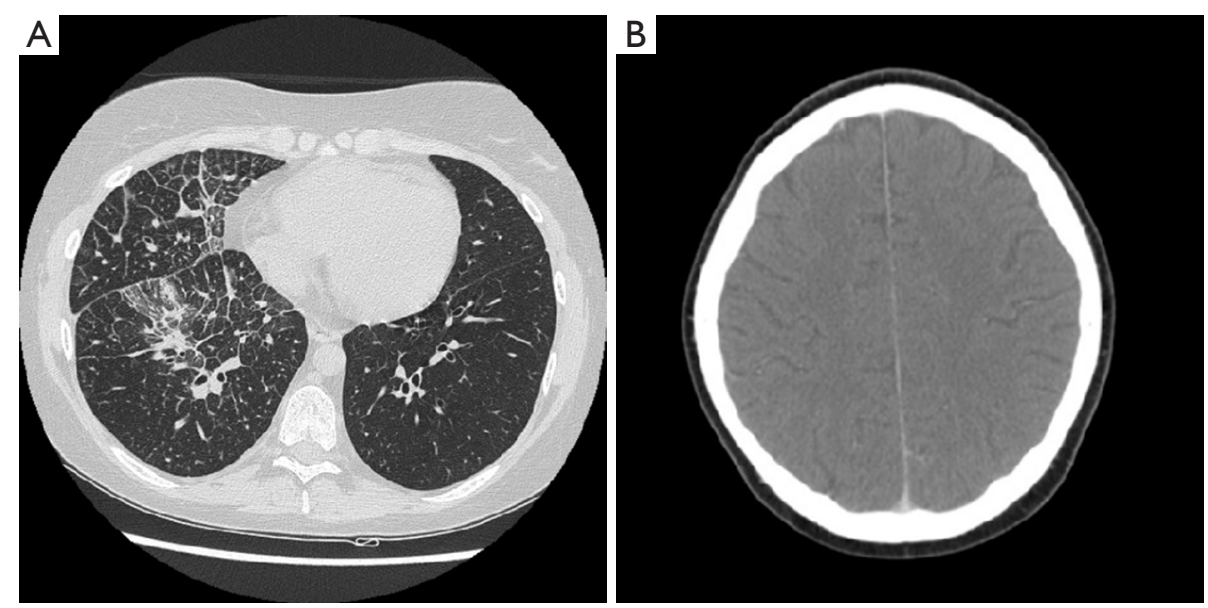

Figure 2 CT scan, January 2015. (A) Chest CT scan; (B) brain CT scan.
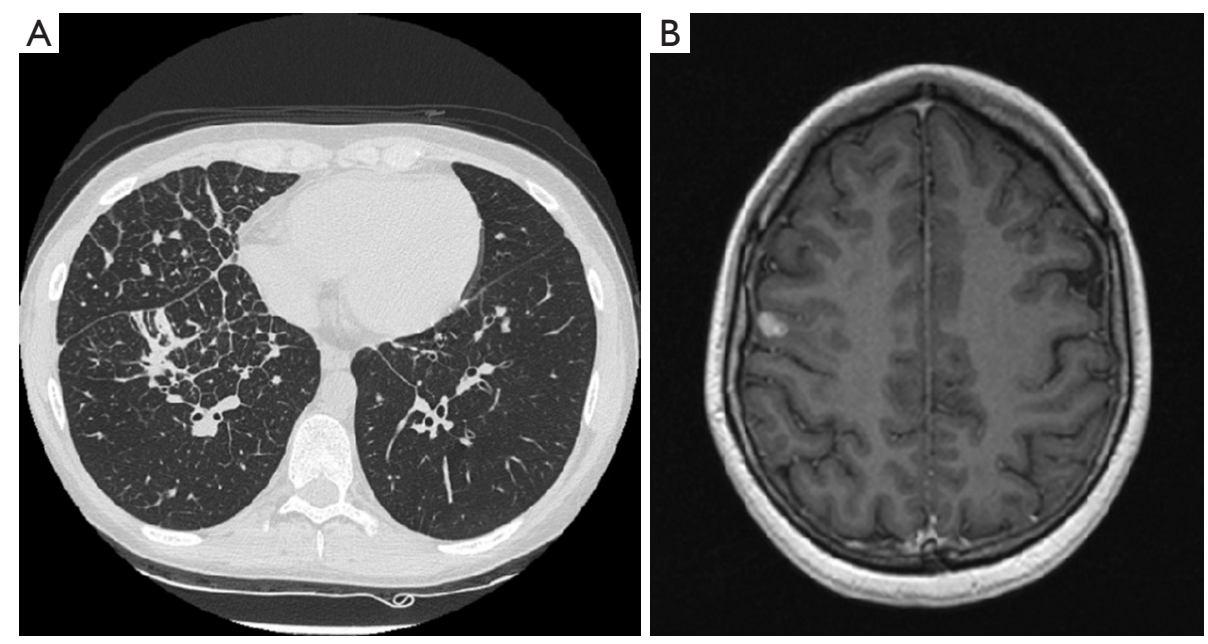

Figure 3 CT scan and MRI, June 2015. (A) Chest CT scan; (B) brain MRI.

tumor proportion score (TPS) $\geq 50 \%$. Currently the patient is being evaluated for lorlatinib compassionate use or for a second line treatment with anti PD-1 monoclonal antibody.

\section{Discussion}

Target therapy for lung cancer patient is an up growing field and ROS1 rearrangement is one of most recently detected targetable gene. Here we describe a dramatic and long-lasting response to Crizotinib treatment in a NSCLC harboring ROS1 rearrangement.

The gender, the young age, the no smoking habit of the patient, the histological and staging features of the disease (mucinous lung adenocarcinoma stage IV at clinical onset) are all NSCLC ROS1+ peculiarities as confirmed by Zhu's meta-analysis (3).

Evidence in literature reported the ROS1 translocations as a crucial event in tumourigenesis of lung adenocarcinoma in young patients. In particular, Scarpino et al. presented 6 of 6 cases (100\%) of lung adenocarcinoma diagnosed in patients $<30$ years of age translocated for $A L K$ (4 cases) or ROS1 ( 2 cases). The six patients $<30$ years of age $(5 \mathrm{~F} / 1 \mathrm{M})$, including two pediatric patients ( $\leq 18$ years old), presented with stage IV disease, were never or light smoker, and had no family history of pulmonary tumours (5).

Approximately $10 \%$ to $25 \%$ of patients with lung cancer are initially diagnosed with brain metastases. In our case, the patient was diagnosed with a miliary brain metastases 


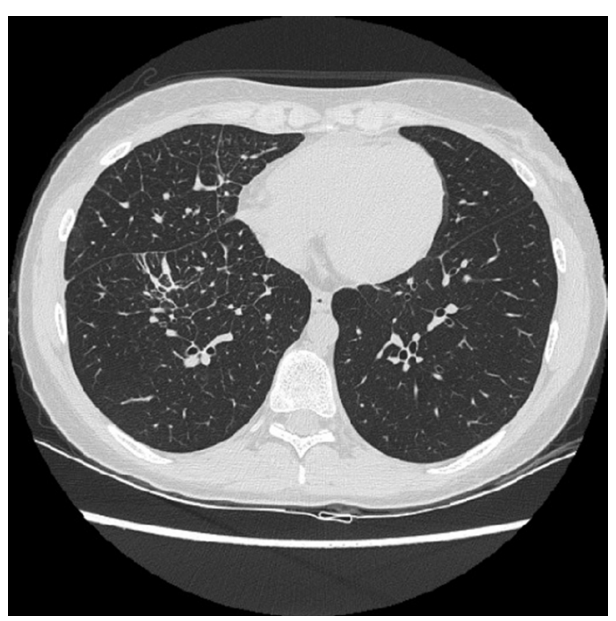

Figure 4 Chest CT scan, October 2016.

dissemination, characterized by the presence of multiple punctate tumor nodules with a perivascular distribution, that is a rare manifestation of brain involvement and often is linked to lung adenocarcinoma. In the presented case the combination of WBRT and crizotinib ensured 7 months of intracranial time to progression (IC-TTP). At the brain oligoprogression, the patient performed a SRS on growing brain lesions and continued the TKI treatment with a long lasting clinical benefit and radiological response. The second IC-TTP occurred after other 16 months. The thoracic radiological evaluation, after the initial partial response, showed a further dimensional reduction of the lung mass, still ongoing with a thoracic progression-free survival (PFS) of 23 months.

To our knowledge, there is only one report in literature of progression in the brain with persistence of response in extracranial sites in NSCLC ROS1 rearranged patients (6). In selected case of oligometastatic progression, integration of Crizotinib treatment with other local therapeutic options such as surgery, radiofrequency ablation, radiotherapy or local ablative therapy could be considered.

Crizotinib is characterized by a low penetration rate to the CSF (0.06-0.26\%) (7). Similar to crizotinib, the $2^{\text {nd }}$ generation ALK inhibitor ceritinib has a limited brain penetration. Conversely, the other $2^{\text {nd }}$ generation ALK inhibitor Alectinib is associated with an intracranial response rate of $52 \%$ (8).

Clinical activity of a $2^{\text {nd }}$ generation ALK TKI, ceritinib, in ROS positive NSCLC has been evaluated in a phase II study, showing, among the 28 evaluable crizotinib naive patients, an ORR of $67 \%$ and a median PFS of 19.3 months with ceritinib treatment (9). In the STARTRK-1 and in the ALKA-372001, entrectinib, a next generation ALK TKI, was well tolerated and active against 14 crizotinib naive patients with ROS1 gene fusions with an ORR of $86 \%$ (10). Ceritinib and entrectinib demonstrated intracranial activity in preclinical studies but they are both unable to overcome ROS1 mutations and other mechanisms of resistance reported in patients relapsed during Crizotinib treatment. Results from single-arm phase I and II studies and retrospective analyses strongly supported that ROS1-targeted therapies are superior to standard first line platinum based chemotherapy. The optimal first line ROS1 TKI and second line TKI sequencing aren't yet defined.

In preclinical (11) and clinical data from phase I/II trial, lorlatinib (PF 06463922), a next generation ALK/ROS1 inhibitor, appeared to efficiently penetrate the blood brain barrier (BBB) with intracranial responses in ROS1+ NSCLC patients and to surmount mechanisms of resistance to $1^{\text {rst }}$ and $2^{\text {nd }}$ generation ALK inhibitors [Solomon BJ, Bauer TM, Felip E, et al. Safety and efficacy of Lorlatinib (PF-06463922) from the dose-escalation component of a study in patients with advanced ALK+ or ROS1+ non-small cell lung cancer (NSCLC). 7 Clin Oncol 2016;34;abstr 9009].

Another drug that is been evaluating in ROS1+ patients is cabozantinib (ClinicalTrials.gov identifiers NCT01639508).

It is unknown whether PD-1/PD-L1 are differently expressed in oncogene addicted NSCLC. In a retrospective cohort of 125 metastatic NSCLC, D'Incecco et al. showed that immune checkpoint PD-L1 expression was high in patients with EGFR mutations and $A L K$ translocations (12). No data for ROS1+ patients and immune checkpoint expression are available so far. Given the high tyrosine kinase domains homology between $A L K$ and ROS1, we could suppose that $R O S 1$ rearrangements may have a potential effect on checkpoint PD-L1 expression, as described for other driver mutations.

\section{Conclusions}

In summary, this case report presents a prolonged PFS in a young patient with an expectation of poor prognosis. It may indicate that the integration of crizotinib with local treatments should be considered in ROS1 NSCLC patients experiencing oligometastatic progression. New ROS1 inhibitors, such as lorlatinib could be a future option in patient with uncontrolled brain disease, but in this case, expressing PD-L1 in more than $50 \%$ of tumour cells, immunotherapy is a valid option. In particular, 
considering the expression of both target gene and PD-L1, the association of target therapy with immune checkpoint inhibitors could represent a valid therapeutic choice.

\section{Acknowledgements}

None.

\section{Footnote}

Conflicts of Interest: The authors have no conflicts of interest to declare.

Informed Consent: Written informed consent was obtained from the patient for publication of this case report and any accompanying images.

\section{References}

1. Rimkunas VM, Crosby KE, Li D, et al. Analysis of receptor tyrosine kinase ROS1-positive tumors in nonsmall cell lung cancer: identification of a FIG-ROS1 fusion. Clin Cancer Res 2012;18:4449-57.

2. Bergethon K, Shaw AT, Ou SH, et al. ROS1 rearrangements define a unique molecular class of lung cancers. J Clin Oncol 2012;30:863-70.

3. Zhu Q, Zhan P, Zhang X, et al. Clinicopathologic characteristics of patients with ROS1 fusion gene in non-small cell lung cancer: a meta-analysis. Transl Lung Cancer Res 2015;4:300-9.

4. Shaw AT, Ou SH, Bang YJ, et al. Crizotinib in ROS1rearranged non-small-cell lung cancer. N Engl J Med 2014;371:1963-71.

Cite this article as: Occhipinti M, Falcone R, Onesti CE, Botticelli A, Mazzuca F, Marchetti P, Lauro S. Crizotinib plus radiotherapy in brain oligoprogressive NSCLC ROS1 rearranged and PD-L1 strong. J Thorac Dis 2017;9(11):E985E989. doi: 10.21037/jtd.2017.09.74
5. Scarpino S, Rampioni Vinciguerra GL, Di Napoli A, et al. High prevalence of ALK+/ROS1+ cases in pulmonary adenocarcinoma of adoloscents and young adults. Lung Cancer 2016;97:95-8.

6. Dziadziuszko K, Szurowska E, Pienkowska J, et al. Miliary brain metastases in a patient with ROS1-rearranged lung adenocarcinoma: a case report. J Thorac Oncol 2014;9:e34-6.

7. Costa DB, Kobayashi S, Pandya SS, et al. CSF concentration of the anaplastic lymphoma kinase inhibitor crizotinib. J Clin Oncol 2011;29:e443-5.

8. Gadgeel SM, Gandhi L, Riely GJ, et al. Safety and activity of alectinib against systemic disease and brain metastases in patients with crizotinib-resistant ALK-rearranged non-small-cell lung cancer (AF-002JG): results from the dose-finding portion of a phase 1/2 study. Lancet Oncol 2014;15:1119-28.

9. Lim SM, Kim HR, Lee JS, et al. Open-Label, Multicenter, Phase II Study of Ceritinib in Patients With Non-SmallCell Lung Cancer Harboring ROS1 Rearrangement. J Clin Oncol 2017;35:2613-8.

10. Drilon A, Siena S, Ou SI, et al. Safety and Antitumor Activity of the Multitargeted Pan-TRK, ROS1, and ALK Inhibitor Entrectinib: Combined Results from Two Phase I Trials (ALKA-372-001 and STARTRK-1). Cancer Discov 2017;7:400-9.

11. Zou HY, Li Q, Engstrom LD, et al. PF-06463922 is a potent and selective next-generation ROS1/ALK inhibitor capable of blocking crizotinib-resistant ROS1 mutations. Proc Natl Acad Sci U S A 2015;112:3493-8.

12. D'Incecco A, Andreozzi M, Ludovini V, et al. PD-1 and PD-L1 expression in molecularly selected non-small-cell lung cancer patients. Br J Cancer 2015;112:95-102. 